\title{
Meaningfulness and the associative phase of paired-associate learning: A methodological consideration'
}

\author{
Rudolph W. Sehulz and Eugene A. Lovelace \\ STATE UNIVERSITY OF IOWA
}

\begin{abstract}
This study shows that Ss who are attempting to locate a given CVC trigram among 10 other trigrams take significantly longer to do so with trigrams of low than with trigrams of high meaningfulness. The implications of this finding for the use of a multiplechoice paired-associate task in determining the effects of meaningfulness on the associative phase are discussed.
\end{abstract}

Introduction The present research was designed to answer the following simple question: Given two sets of 10 CVC trigrams with the trigrams of one set high in meaningfulness (M) and those of the other set of low M, how long does it take $S$ to locate a prescribed trigram in each of the respective sets? While the question is a simple one, the reason for seeking an answer to it is not as easily stated and, therefore, will require a brief digression.

It is well established that variation of response-term $M$ has a greater effect on the learning of verbal paired associates (PA) than a comparable variation in the $M$ of stimulus terms (e.g., Cieutat et al., 1958; Underwood \& Schulz, 1960). From both an empirical and a theoretical standpoint, an important next step in the analysis of the role of $\mathrm{M}$ in PA learning is the one of determining the relative contributions of response-term $M$ to the facilitation of learning in the response-learning and associative phases of PA learning. A multiple-choice PA task seemed best suited for the assessment of the effects of response-term $\mathrm{M}$ on the associative phase because it would, at least ostensibly, eliminate response-learning factors by giving $\mathrm{S}$ direct access to the responses on test trials. If under these conditions the asymmetry in M's effectiveness on the stimulus vs. the response sides of a PA list were to be absent, it could then be inferred that this asymmetry is due entirely to the effect of responseterm $\mathrm{M}$ on the response-learning phase.

In our first experiment (Schulz \& Hart, 1962, unpublished), Ss were presented with alternating study and test trials with a PA "mixed-list" version of a $2 \times 2$ design for stimulus vs. response $M$. On test trials all responses appeared each time the stimulus of a given pair was presented. Thus S did not need to recall responses, merely find them and read them off. The results obtained with this procedure were not very different from those obtained with the standard anticipation procedure except that response M probably had slightly less effect. Had that attempt to eliminate all response-learning factors been successful? Evidently not, an analysis of Ss errors re- vealed clear evidence that Ss might have recalled high M units but looked for low M units. This source of contamination was removed in a second experiment (Schulz \& Carlson, 1962, unpublished) by requiring Ss to press a button to indicate his choice, thus forcing all Ss to locate the response term of test trials. Nevertheless, the effects from response $M$ were again found to be greater than those of stimulus $M$. This result agreed with past findings, but not with our expectations. Moreover, having been alerted to possible artifacts in the present procedure by our first experiment, our wariness regarding the presence of additional artifacts and the latency data of the preceding experiment led us to consider the possibility that the greater effect of response-term $M$ in this experiment may have resulted from Ss having required less time to locate high $M$ than low $M$ responses. Hence, the question: Does the latency of S's location-response vary as a function of $\mathrm{M}$ in a multiple-choice PA task?

Method Four lists of $10 \mathrm{CVC}$ trigrams, the same ones used by Schulz and Carlson (1962, unpublished), were used in the present study. There was one set of two high $\mathrm{M}$ lists and one set of two low M lists. According to Archer (1.960), the items in the high $M$ set had an average $\mathrm{M}$ of $96.7 \%$ while the average $\mathrm{M}$ of the low $\mathrm{M}$ set was $20.4 \%$. Intralist formal similarity, in terms of number of duplicated letters, was low in all lists.

The Ss, 40 students in introductory psychology at the University of Iowa, were assigned to a list at random with the restriction that the Nth $\mathrm{S}$ not be assigned to a given list until N-1 Ss had been assigned to each of the other lists. The lists were presented on a modified memory drum. The aperture of the memory drum was divided by a thin strip of black cardboard into a 1 in stimulusaperture (SA) at the left and an 8 in response-aperture (RA) to the right. Ten white pushbuttons were equally spaced horizontally 1 in below RA. Each button was associated with a light on E's side of the drum and indicated S's choice. A trial, the analogue of a test-trial in multiple-choice PA learning, consisted of one presentation of each of the 10 trigrams in a given list in SA with each of the 10 trigrams, including the one in SA, being displayed in RA each time a different trigram was presented in SA. The Ss were instructed to find, as rapidly as possible, the trigram in SA among those presented in RA and to push the button below the appropriate trigram. The latency of S's responses was measured with a Model 120A Hunter KlocKounter which was activated by the appearance of a trigram in SA and stopped when S 


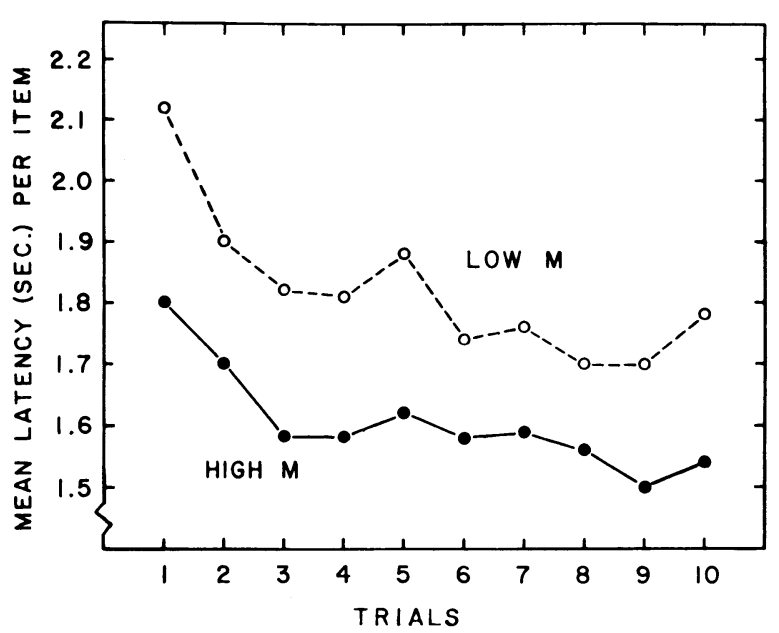

Fig. 1 .

depressed a button. The trigrams in RA were positioned so that a button below RA was associated with each trigram. There were five random orders for the appearance of a trigram in SA and with respect to the position of a trigram in RA. The exposure duration of each trigram array was $5.2 \mathrm{sec}$. The inter-array interval was $0.8 \mathrm{sec}$. The intertrial interval was $6 \mathrm{sec}$.

Results and Discussion The answer to the question raised at the outset of this paper is shown in Fig. 1. There it can be seen that the mean location-latencies (sec.) per item are clearly longer for low than for high items. It is also apparent that the latencies decrease as a function of trials for both types of items. Statistically, the differences in performance both as a function of $\mathrm{M}(\mathrm{F}=8.37$, df $1 / 38, \mathrm{P}<.01)$ and trials $(\mathrm{F}=12.45$, df $9 / 342, \mathrm{P}<.01)$ are highly reliable sources of variance while the interaction of $M$ and trials failed to approach significance $(\mathrm{F}<1)$.

In light of the present results, it seems quite evident that differences in location-latency must be considered a potential source of bias in the investigation of the effects of response-term $M$ on the associative phase of PA learning when a PA task with multiple-choice test-trials is employed. It remains, of course, to be determined whether ways can be found to eliminate this artifact or take it into account. Several possibilities suggest themselves e.g., preliminary practice, increasing the length of the choice interval on test-trials, reducing the number of alternatives. Of these possibilities, the last one seems, for reasons whose consideration is precluded by lack of space, most promising. Indeed, though the dissyllables provided by Noble (1952) and not the present trigrams were involved, preliminary analyses of the data from a study recently completed in our laboratory failed to reveal differences in location-latency as a function of Noble's $M$ when only four alternatives were presented on test trials. Nevertheless until detailed data are available regarding the latencies of location-responses under various conditions, it would seem mandatory that investigators pre-test their materials by means of a study similar to the present one before undertaking studies of the associative phase of PA learning with the multiple-choice technique.

Finally, methodological considerations aside, it is of interest to speculate briefly as to why it took Ss longer to locate low than high M trigrams in the study. Since formal intralist similarity was carefully controlled, it cannot be considered a factor. Nevertheless, differential discriminability or ease of recognition as a function of M would seem a plausible interpretation. Particularly so, in light of the support such an interpretation receives from recent demonstrations that Noble $M$ and tachistoscopically determined visual duration thresholds covary, thresholds being lower for high than for low M dissyllables (e.g., Kristofferson, 1957; Johnson et al., 1961). Similarly, Spence (1963) has provided evidence that given only partial cues to a word's identity at varying duration levels, familiar words were recognized more rapidly than unfamiliar ones. On the other hand, Taylor (1958), working with CVC trigrams, failed to find a relationship between $\mathbf{M}$ and duration threshold.

An alternative, though perhaps not unrelated, explanation is suggested by Conrad's (1962) finding that, among other things, an inverse relationship exists between reading rate and the $\mathrm{M}$ of $\mathrm{CVC}$ trigrams. Namely, $\mathrm{S}$ having had less prior experience, as inferred from Underwood and Schulz's (1960) demonstration that the M of CVC trigrams and their frequency of occurrence in the language is directly related, in reading low than high $\mathbf{M}$ trigrams, it takes him longer to read them and in turn the latency of his location-response must inevitably be increased. Obviously, the present data fail to provide grounds for a decision between these alternative interpretations. Such a decision must await further research.

\section{Referenees}

ARCHER, E. J. A re-evaluation of the meaningfulness of all possible CVC trigrams. Psychol. Monogr., 1960, 74, No. 10 (Whole No. 497). CIEUTAT, V. J., STOCKWELL, F. E., \& NOBLE, C. E. The interaction of ability and amount of practice with stimulus and response meaningfulness $\left(m, m^{\prime}\right)$ in paired-associate learning. J. exp. Psychol., 1958, 56, 193-202.

CONRAD, R. Practice, familiarity and reading rate for words and nonsense syllables. Quart. J. exp. Psychol., 1962, 14, 71-76.

JOHNSON, R. C., THOMSON, C. W., \& FRINCKE, G. Word values, word frequency, and visual duration threshold. Psychol. Rev., 1960, $67,332-342$.

KRISTOFFERSON, A. B. Word recognition, meaningfulness, and familiarity. Percept. mot. Skills, 1957, 7, 219-220.

NOBLE, C. E. An analysis of meaning. Psychol. Rev., 1952, 59, 421-430. TAYLOR, J. A. Meaning, frequency, and visual duration threshold. J. exp. Psychol., 1958, 55, 329-334.

SPENCE, JANET TAYLOR. Contribution of response bias to recognition thresholds. J. abn. soc. Psychol., 1963, 66, 339-344.

UNDERWOOD, B. J., \& SCHULZ, R. W. Meaningfulness and verbal learning. Chicago: Lippincott, 1960.

\section{Note}

1. The authors are indebted to Mr. Richard Vardaris for collecting the data reported in the present paper and for helping with their analysis. 